\title{
Pain treatment satisfaction in spinal cord injury
}

\author{
D Murphy*,1,2 and DB Reid ${ }^{1,2}$ \\ ${ }^{1}$ Woodrow Wilson Rehabilitation Center, Fishersville, Virginia, USA; ${ }^{2}$ University of Virginia Health Sciences Center, \\ Department of Physical Medicine and Rehabilitation, Charlottesville, Virginia, USA
}

Study design: A survey on pain satisfaction was mailed to 300 individuals with spinal cord injury. Eighty-eight completed surveys were returned, and the results were analyzed.

Objectives: The survey queried the respondents on characteristics of their pain, treatments received, the impact of pain on multiple, life activities and functions and the satisfaction with treatment received to reduce pain.

Setting: Subjects for the study were selected from the Spinal Cord Injury Registry from the Commonwealth of Virginia in the US.

Methods: Information was obtained from a survey sent to the subjects who were chosen randomly with respect to age and gender. At least 1 year had elapsed from the time of injury for each individual.

Results: Respondents were typically dissatisfied with the results of the treatments received to manage their pain.

Conclusion: Pain in individuals with spinal cord injury needs to be addressed in a thorough fashion to reduce the adverse impact on life activities.

Spinal Cord (2001) 39, 44-46

Keywords: Spinal cord injury; pain management; rehabilitation; treatment satisfaction

\section{Introduction}

Pain commonly occurs in individuals with spinal cord injury (SCI). The reported incidence and prevalence after spinal cord injury shows great variation due to the difficulties in terminology and definitions. ${ }^{1}$ Mariano $^{2}$ reviewed estimates of the prevalence of severe/ disabling chronic pain in SCI and the numbers ranged from $18 \%$ to $63 \%$. Demirel et $a l^{3}$ studied 47 individuals with SCI and found that $61 \%$ had moderate to severe pain. Shoulder pain and dysesthetic pain are some of the more common types of physical discomfort typically associated with quadriplegia. Nichols et al surveyed individuals with SCI living in the community and found $50 \%$ of respondents experienced shoulder pain. Numerous different types of therapies have been utilized to either eliminate or reduce this type of pain in this patient population. Specifically, physical modalities such as heat and cold treatments, medications such as non-steriodal antiinflammatory and narcotic medications, and various types of shoulder injections have been tried to ameliorate shoulder pain. For dysesthetic pain additional measures have included anticonvulsants and antidepressant medications. Sufferers are frequently referred to multidisciplinary pain clinics for treatment. Patients and clinicians often experience frustration in achieving satisfactory control of these pain syndromes. The authors conducted a survey of

*Correspondence: D Murphy, Woodrow Wilson Rehabilitation Center, Fishersville, Virginia 22939, USA individuals with SCI to determine levels of satisfaction with the treatment methods available. Satisfaction with treatment is of course a highly individual consideration and each individual will define satisfactory treatment with individual differences. The authors did not attempt to preassign criteria for expressing satisfaction but rather allowed the respondent to express, from what naturally has to be a very personal viewpoint, whether or not the given individual felt satisfied with their pain management. The clinician and the patient may hold opposing viewpoints on this issue. Reasonable time had passed for pain management access and interventions to occur for all respondents to the survey. The survey did attempt to some degree to ascertain what portion of available treatments the respondent had received. The information gathered was through self-report. All levels of injury were verified through the spinal cord registry of the Commonwealth of Virginia. In the cases of a discrepancy the information from the registry was used.

\section{Methods}

\section{Subjects}

The subjects were chosen randomly with respect to age and gender from the spinal cord injury registry of the Commonwealth of Virginia. All subjects had a spinal cord injury which was either incomplete or complete. Only individuals in the chronic stages of their injury 
(more than 1 year after the date of injury) were chosen for participation.

\section{Procdeure}

Questionnaires were either mailed or handed out to 300 potential participants. One hundred and three were returned. Fifteen were incomplete and consequently discarded. Eighty-eight were complete. The survey specifically focused upon shoulder and dysesthetic pain with inquiries into pain duration and intensity, types of treatments received, efficacy of treatments, satisfaction with treatments, and the impact of pain on the individual's life. The questionnaire did not attempt to differentiate the multiple, potential causes of shoulder pain (tendonitis, bursitis, trigger point pain, neuropathic pain, etc.) yet focused on shoulder pain because of its greater potential impact on functional activities. Dysesthetic pain was defined for the respondent as pain attributed by their physician to the injury to the spinal cord.

\section{Results}

The mean age of respondents was 42 years. Sixty-nine per cent were males and $16 \%$ females. The onset of injury ranged from 1956 to 1998. Seventy-two respondents had cervical injuries and one a mixed cervical/thoracic level injury. Fifteen respondents did not indicate a level of injury. Seventy per cent of respondents reported shoulder pain, while $74 \%$ reported dysesthetic pain. Of those with shoulder pain, $70 \%$ indicated they had pain for 6 months or longer. For those with dysesthetic pain, 74\% had pain 6 months or longer. A majority of subjects reporting pain received some form of treatment for their pain, which most frequently involved ibuprofen, hot packs and narcotics. In general, treatment for shoulder pain was perceived as more effective than treatment for dysesthetic pain. Relatively few subjects received treatment in a chronic pain program, but most of those who did believed the pain program was either ineffective or rarely effective. Sixty-seven per cent of subjects reporting shoulder pain indicated specific adverse effects on their lives with the most frequent areas being recreation, self-care activities and social life. Sixty-five per cent of those reporting dysesthetic pain indicated specific adverse effects primarily with recreation and social activities. The impact of pain on life in general was rated as important or very important in $57 \%$ of cases. Forty per cent of respondents with shoulder pain rated their worst pain as 7 or higher on a scale of one to ten ( $1=$ no pain; $10=$ worst possible pain). On the same scale, $57 \%$ of those with dysesthetic pain rated their worst pain as 7 or higher. However, only $19 \%$ were satisfied with the medical treatments received. Fortynine per cent were dissatisfied, and $32 \%$ did not respond to the question.

\section{Discussion}

Most respondents in this study reported shoulder pain and dysesthetic pain that could be rated severe at its worst and that frequently lasted 6 months or longer. The most frequently mentioned treatments were ibuprofen, narcotic medications and hot packs. The majority of respondents reported dissatisfaction with the results of the management of their pain. This lack of adequate pain control could have several possible explanations. Treatment programs for these individuals may not have adequately explored all of the options. Perhaps these respondents represent a subset of spinal cord injured individuals whose shoulder and/or dysesthetic pain comprises a type that is refractory to any of the current methods of pain management. From the survey, it appears that not all avenues were adequately explored. Hence, one can not draw any firm conclusions concerning treatment efficacy in SCI among the subjects in this survey. Many of these individuals had longstanding injuries and thus from a time perspective had ample opportunity to explore a wide spectrum of pain management options. Since these subjects reported substantial impact from pain on their personal lives, one could ask why further treatments were not explored. Reasons might include those stemming from the individual with SCI and those stemming from the individual's health care delivery system. The list of possibilities is extensive and would include patient motivation and compliance, reimbursement systems, the level of interest and knowledge on the part of health care providers, transportation difficulties, etc. Some of the respondents indicated that the treatment methods either did not work or did not work long enough for them to consider any of the options tried with them as successful. Out of the wide array of pain management options available (e.g. physical or occupational therapy interventions, medications including antidepressants, topical agents, antispasticity agents, antiarrhythmics, nonnarcotic analgesics, narcotics and anticonvulsants, injection techniques, and even perhaps surgery), only a small fraction were tried by any of the respondents in this survey. Some options not mentioned include acupuncture and either baclofen or morphine pumps or potentially intrathecal clonidine. ${ }^{5}$ Dalyan et $a l^{6}$ emphasized wheelchair or home modifications and joint protection education for upper extremity pain after SCI. Fenollosa et $a l^{7}$ reported on the treatment of 33 patients using various combinations of medications (amitriptyline, clonazepam, NSAIDs, 5-OH-tryptophane), spinal cord stimulation, and morphine by continuous intrathecal infusion. They reported that analgesia reached $80 \%$ in all cases. More recently, Eide $e t \quad a l^{8}$ successfully employed ketamine and alfentanil in the reduction of SCI dysesthetic pain. They concluded that central dysesthetic pain in SCI is dependent on activation of central NMDA 
receptors. Also, mu-opioid receptors can play a role in the control of this pain.

A confounding factor to the interpretation of this study is the status of those who did not respond to the survey. It is conceivable that non-respondents had less pain or perhaps more successful treatment of their pain. One could also question the accuracy with which this small sample represents the spinal cord injury population at large. Furthermore, it is unknown if Virginia residents with SCI have the same range of pain therapies available to them as individuals from other states. More extensive and widely-based surveys are required for these concerns to be answered adequately.

As research in pain management progresses, the options for control of SCI-related pain continue to improve. The full spectrum of pain treatments needs to be applied to this patient population since pain can substantially and adversely affect one's quality of life; only then can a widely-based survey indicate the success of current treatment methods.

\section{References}

1 Siddal PJ, Taylor DA, Cousins MJ. Classification of pain following spinal cord injury. Spinal Cord 1997; 35: 69-75.

2 Mariano AJ. Chronic pain and spinal cord injury. Clin J Pain 1992; 8: $87-92$.

3 Demirel G, Yllmaz H, Gencosmanoglu B, Kesiktas N. Pain following spinal cord injury. Spinal Cord 1998; 36: 25-28.

4 Nichols PJR, Norman PA, Ennis JR. Wheelchair user's shoulder? Shoulder pain in patients with spinal cord lesions. Scand J Rehab Med 1976; 11: $29-32$.

5 Middleton JW et al. Intrathecal clonidine and baclofen in the management of spasticity and neuropathic pain following spinal cord injury: a case study. Arch Phys Med Rehab 1996; 77: $824-$ 826.

6 Daylan M, Cardenas DD, Gerard B. Upper extremity pain after spinal cord injury. Spinal Cord 1999; 37: 191-195.

7 Fenollosa et al. Chronic pain in the spinal cord injured: statistical approach and pharmacological treatment. Paraplegia 1993; 31: $722-779$.

8 Edie PK, Stubhaug A, Stenehjem AE. Central dysesthetic pain after traumatic spinal cord injury is dependent on N-methyl-Daspartate receptor activation. Neurosurgery 1995; 37: 10801087. 